\title{
Application of Classical Oil Painting Techniques in Chinese Contemporary Lacquer Painting Creation
}

\author{
Ran Li \\ Eastern International Art College \\ Zhengzhou University of Light Industry \\ Zhengzhou, China
}

\begin{abstract}
Chinese contemporary lacquer painting must have sturdy basic skill, be creative, learn other useful theories and enrich internal culture of lacquer painting to improve painting. Modern lacquer art requires exquisite making and keeping improving. Contemporary aesthetic characteristics need to be displayed in creation. Painting is the basis of contemporary lacquer painting. For a long time, most Chinese lacquer painters attach great importance to craft, but their painting skills are not good enough. Preciseness in modeling and aestheticism and elegance of classical oil painting techniques make up for painting of contemporary lacquer painting creation.
\end{abstract}

Keywords-classical oil painting; contemporary lacquer painting; creation; application

\section{INTRODUCTION}

In contemporary Chinese lacquer painting creation, no matter it is adoption of decoration method or painting technique, or development of new techniques, new crafts and new ideas, or application of various kinds of techniques, as long as it starts from aesthetic appreciation of contemporary lacquer painting creation, only by using "painting" to implement, insist and innovate, can it break through constriction of traditional lacquer art and make progress in the field of contemporary lacquer painting. All painters who are perfect in mastering lacquer art are good at researching lacquer art and combining it with painting. They use the form of "painting" to create and make true contemporary oil paintings. Preciseness in modeling and aestheticism and elegance of classical oil painting techniques make up for painting of contemporary lacquer painting creation.

\section{RESEARCH STATUS OF APPLICATION OF CLASSICAL} OIl PAINTING TECHNIQUES IN CONTEMPORARY CHINESE OIL PAINTING CREATION AT HOME AND ABROAD

Foreign people see lacquer painting art as decoration art or products of artware, and they do not admit its nature of painting creation. Our lacquer painters should strive to use their own creation practice to display superb painting techniques of lacquer painting, thus "developing a school of our own" in arts circle of the world. Therefore, we need to create more better lacquer painting works and carry forward.
Now various art schools list lacquer painting in their department of arts and crafts. Actually, lacquer painting is a kind of painting. Chinese artists association establishes lacquer painting art committee in 2001 and lists lacquer painting in painting. Since we admit the nature of painting of contemporary lacquer painting, we should carry out according to painting creation rule; paintings should reflect life, move people with their connotation and aestheticism and have classical beauty like oil painting; they should also have painting beauty of material, technique and tool, meanwhile, we can use transparent or semitransparent glazing of classical oil painting to guide lacquer painting's colored painting technique of rendering. These two have a lot in common as to techniques, therefore contemporary lacquer painting art can be expressed vividly by using strong painting form.

Lacquer is a symbol of Chinese culture and it is a national feature based on seven thousand years' profound historical culture. From 1980s to the 21 st century, lacquer is displayed in former Soviet Union, Japan, South Korea, Vietnam and Europe on behalf of Chinese culture and wins a lot of love. Chinese lacquer art has agelong historical resources as basis. As a new subject, it gradually wins attention from academic lacquer painting creator during its unceasing development process. In the past 40 years, China's eight great colleges of fine arts, Nanjing University of the Arts, Fuzhou Normal University's Department of Art and Crafts etc. list lacquer painting as decoration art or industrial art, and they do not think that lacquer painting has enough nature of painting. Seeing from the 10th national fine arts exhibition's lacquer painting works in 2004, the 11th in 2009 and the 12th in 2014, lacquer painting has more and more painting nature and classical oil painting's delicate glazing technique can be found everywhere in today's lacquer painting depicting performance techniques.

\section{INNOVATIVE APPLICATION OF CLASSICAL OIL PAINTING TECHNIQUES IN CONTEMPORARY CHINESE LACQUER PAINTING CREATION}

Classical oil painting has a long history with skilled techniques and it can direct or guide innovative development of other arts in the field of art modeling. Contemporary lacquer painting starts in international art circle in 1940s and it is not mature enough in drawing techniques. Through 
research on materials of classical oil painting and contemporary lacquer painting, we find that: Classical oil painting and contemporary lacquer painting have some similarities in color mixing medium and diluent. Classical oil painting uses oil-based medium to mix color to make the picture smooth and gentle while contemporary lacquer painting can use both Chinese lacquer and boiled tung oil to mix paint and do painting. By using mixture of boiled tung oil and toner to trace the oil and do colored drawing, the picture can also be gentle and gradually change. You can also mix Chinese lacquer and toner together and add an appropriate amount of boiled tung oil, then do painting. This technique emphasizes on rendering. Seeing from this aspect, classical oil painting and contemporary lacquer painting has certain similarities. The former emphasizes on glazing and the latter emphasizes on tendering. Although they have different names, their technique principles are the same. They both contain the process of oily dyeing gradient. In this way, both classical oil painting and contemporary lacquer painting can produce rigorous modeling and be gentle and smooth at the same time. Both of them can be freely controllable in techniques in terms of lightness, purity, hue and light and shade gradient. They both can use turpentine to clean picture in terms of diluent. Therefore, they two have some similarities.

\section{CONTEMPORARy CHINESE LACQUER PAINTING CREATION NEEDS ClassiCAL OIL PAINTING'S GLAZING TECHNIQUE}

Contemporary lacquer painting is restricted in material, technique and creation process. Actually, restriction of material is not the main part. The main question is how to overcome drawing compatibility of Chinese lacquer and toner and how to correctly use boiled tung oil colored drawing method. Only by doing this, can we overcome various restrictions on materials. We can obtain progress by being good at groping and keeping forging ahead. In addition, highly prosperous decorative style of Ming and Qing Dynasties has great influence on contemporary lacquer painting. Now, many colleges of fine arts list lacquer painting in department of arts and crafts and training of basic skill of sketch and realistic painting is neglected in colleges. All these hinder development of contemporary lacquer painting's painting nature largely. In fact, these restrictions make lacquer painting bad at detailed depicting of object and paintings fail to depict features reflecting object's original artistic appearance. In many colleges of fine arts, a lot of teachers and students are blindly painting small flowers, small scenery or small subject and small scope. Their painting excessively displays decorative style or texture characteristics and loses real feature of art. Moreover, contemporary art creation excessively imitates western modernist school and displays rough brushwork and abstractness, making contemporary lacquer painting bad at truthfully depicting object enters another wrong region of creation. It just strives to demonstrate and emphasize sense of beauty of texture resulted from author's embossed lacquer, craved lacquer, scribed line, inlay and polishing. Although it looks brighter than oil painting in color and richer than traditional Chinese painting in texture, and it has more woodcut nature than wood block, more decorative concaveconvex sense than ornamental sculpture and more ceramics flowers than chinaware's surface, all these are just superficial phenomena. In fact, lacquer painting has painting and craft nature. The so-called $70 \%$ painting and $30 \%$ craft is the original appearance of lacquer painting. Painting nature occupies a larger part, so we can say painting nature is the soul of lacquer painting. And craft nature also belongs to painting nature. Today's colleges of fine arts should not list lacquer painting in department of arts and crafts or department of decoration art. First, their teaching positioning neglects painting nature. They should list lacquer painting as drawing major to recruit students from the whole country. Catch up from basis and basic skill to lay foundation for lacquer painting's painting style. Seeing from lacquerware works in Han and the North Wei Dynasties, we can know that painting nature is extremely prominent in that period. The single colored drawing technique artists only have in that period can produce magnificent works. All these should be excellent examples for contemporary lacquer painting to learn and use for reference.

In conclusion: Contemporary lacquer painting's nature of painting is restricted by human reasons or natural materials, thus making contemporary lacquer painting lose its soul in painting nature and making it hard to put to good use. We can learn experience and lessons from it. We can learn classical oil painting's rigorous scholarship and spirit of not giving up the goal. Classical oil painting can lead contemporary lacquer painting's painting technique in transparent or semitransparent glazing technique. Meanwhile, contemporary lacquer painting can refer to or learn from western classical oil painting technology in painting. Contemporary lacquer painting can use the advantage of classical oil painting to make up for its imperfection in painting creation. Of course, learning can not be just about free riding or following the trend to learn modernism art.

\section{Classical Oil Painting TeChniques Can MaKe UP FOR LIMITATION IN CONTEMPORARY CHINESE LACQUER PAINTING CREATION}

Classical oil painting appears in the 14th century's Europe and prospers in the 16th century's European renaissance in Italy. Classical oil painting technique reaches its peak in the middle of 18th century and its performance practice is totally different from direct drawing method of later oil painting. The difference is that it conducts glazing layer by layer among semitransparent and transparent color layers and then overlays them to display quality-shaped and rigorous modeling, which is called indirectly painting. Through repeatedly transparent or semitransparent painting, an indirectly painted profound classical oil painting comes out with implicit, rich, perfect, rigorous, refined and gentle style characteristics. This method has been used from 14th century to the beginning of 18th century in Europe. Although classical oil painting only has 50 years' brilliant achievement in China, Chinese classical oil painting has some influence in international art circle in technique and connotation. 


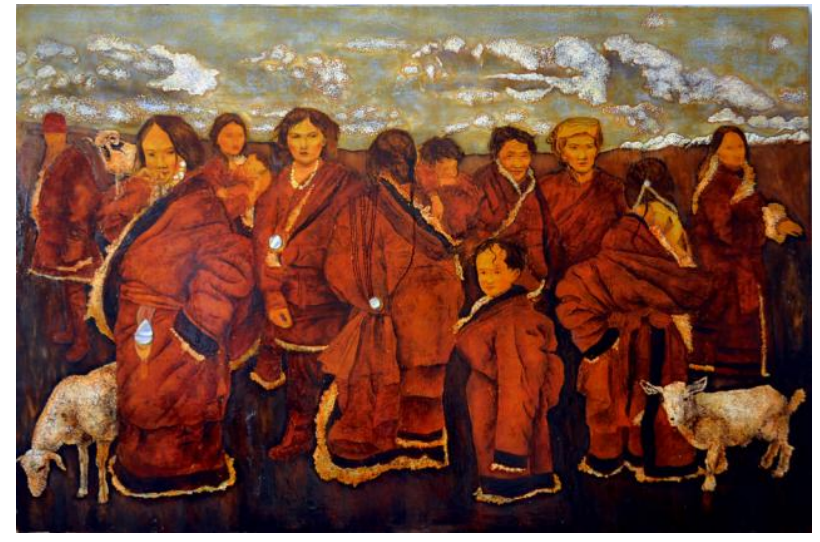

Fig. 1. Contemporary lacquer painting "Altitude: 4700 meters"

Contemporary lacquer painting develops to nowadays from the basis of ancient lacquerware "Fig. 1". Although ancient lacquerware has a history of 7000 years, contemporary lacquer painting only has a history of 50 years in China. Therefore, it is not as time-honored as European classical oil painting in performance techniques and not as mature as European classical oil painting technique and there is still improving space. As to material, classical oil painting mainly uses oil paint while contemporary lacquer painting uses power paint. Although they use different pigments for painting, they have some similarities in color mixing medium and diluent. Classical oil painting uses oily medium to mix color, making the picture smooth and gentle. Contemporary lacquer painting uses Chinese lacquer to mix paint and it can also use boiled tung oil to mix paint and do painting. Mix boiled tung oil and toner together and add an appropriate amount of transparent Chinese lacquer to trace the oil and do colored drawing, realizing gentle gradient of the picture. The technique emphasizes on tendering. Considering from this aspect, classical oil painting and contemporary lacquer painting has some similarities. The former emphasizes on glazing and the latter emphasizes on tendering. Although they have different names, they are the same in technique principle. They both contain oil dyeing gradient process. In this way, both classical oil painting and contemporary lacquer painting can get rigorous modeling. Both of them can be freely controllable in techniques in terms of lightness, purity, hue and light and shade gradient. They both can use turpentine to clean picture in terms of diluent. Although they two have some similarities, classical oil painting technique is more rigorous and more delicate. Altitude 4700 Meters is a lacquer painting works of mine in 2013. It uses classical oil painting's glazing technique and boiled tung oil and transparent Chinese lacquer as material. I spend 5 months in my studio creating the contemporary lacquer painting Altitude 4700 Meters with classical oil painting techniques. The Tibetan people's face, hands and costume texture use boiled tung oil colored drawing method. Detailed parts of skin color are soft and gentle. They indirectly and partially use classical oil painting's glazing technique for reference. Remaining parts of the picture use art decoration method. The accessories on Tibetan women's body use eggshell or shell inlay technique. Inlay part uses density variable and gradual change of empty and full.
Primary color of inlay part after polishing or coloring and glazing makes the whole picture harmonious in color with graceful and elegant features, lightening out contemporary lacquer painting's charming language. Practice has proved: Classical oil painting technique can make up for Chinese contemporary lacquer painting's painting nature very well.

\section{CONCLUSION}

Although classical oil painting and contemporary lacquer painting are two different kinds of painting. They are different in material, but classical oil painting and contemporary lacquer painting have some similarities in glazing or tendering technique. Classical oil painting can use its glazing technique to guide contemporary lacquer painting's painting technique. Meanwhile, contemporary lacquer painting can refer to or learn from western classical oil painting techniques in painting nature. There is still space for improvement for contemporary lacquer painting's development of future painting technique.

\section{REFERENCES}

[1] Qiao Shiguang Lacquer Painting Techniques and Artistic Expression, China Hunan Fine Arts Publishing House, 1996

[2] Sun Hongtu, Jin Chengbin, Wu Jiaquan: Lacquer Painting Tutorial, Liaoning Fine Arts Publishing House, 2010-03

[3] Liu Chunchao "Lacquer painting's painting nature" The 6th Issue of Guangdong Art, 2009

[4] Qiao Shiguang: Lacquer Art, Hangzhou, China Academy of Art Publishing House, 2000.

[5] Su Guowei: "By-talk on lacquer painting's painting nature and contemporariness" The 16th Issue of Popular Literature, 2010 